\title{
ON LIPSCHITZ FUNCTIONS OF NORMAL OPERATORS
}

\author{
FUAD KITTANEH
}

\begin{abstract}
It is shown that if $N$ and $M$ are normal operators on a separable, complex Hilbert space $H$, and $f$ is a Lipschitz function on $\Omega=\sigma(N) \cup \sigma(M)$ (i.e., $|f(z)-f(w)| \leqslant k|z-w|$ for some positive constant $k$ and all $z, w \in \Omega$ ), then $\|f(N) X-X f(M)\|_{2} \leqslant k\|N X-X M\|_{2}$ for any operator $X$ on $H$. In particular, $\|f(N)-f(M)\|_{2} \leqslant k\|N-M\|_{2}$
\end{abstract}

Let $H$ denote a separable, complex Hilbert space and let $B(H)$ denote the algebra of all bounded linear operators acting on $H$. An operator $T \in B(H)$ is said to belong to the Hilbert-Schmidt class $C_{2}$ in case $\sum_{i, j}\left|\left(T e_{i}, e_{j}\right)\right|^{2}=\Sigma_{i}\left\|T e_{i}\right\|^{2}$ is finite for some (hence, for all) complete orthonormal systems $\left\{e_{i}\right\}$ in $H$. For $T \in C_{2}$, let $\|T\|_{2}=\left(\sum_{i}\left\|T e_{i}\right\|^{2}\right)^{1 / 2}$ be the Hilbert-Schmidt norm of $T$. The properties of HilbertSchmidt operators are described in Schatten [9] and Gohberg-Krein [7].

In their work on Scattering theory, W. O. Amrein and D. B. Pearson proved [1, Theorem 2] that if $A$ is a selfadjoint operator with pure continuous spectrum and $f$ is a Lipschitz function on $\sigma(A)$ (the spectrum of $A$ ) i.e., $|f(t)-f(s)| \leqslant k|t-s|$, then $\|f(A) X-X f(A)\|_{2} \leqslant k\|A X-X A\|_{2}$ for all $X \in C_{2}$. Utilizing Voiculescu's perturbation property of normal operators [10], we now establish the following considerable generalization of the Amrein-Pearson result.

THEOREM. Let $N$ be a normal operator. Let $f$ be a function defined on $\Omega=\sigma(N)$. If $|f(z)-f(w)| \leqslant k|z-w|$ for all $z, w \in \Omega$ and some positive constant $k$, then

$$
\|f(N) X-X f(N)\|_{2} \leqslant k\|N X-X N\|_{2} \text { for all } X \in B(H) \text {. }
$$

Proof. Given $\varepsilon>0$, let $N=D_{\varepsilon}+K_{\varepsilon}$, where $D_{\varepsilon}$ is diagonal and $\left\|K_{\varepsilon}\right\|_{2}<\varepsilon$ [10]. If $D_{\varepsilon} e_{n}=\lambda_{n} e_{n}$ and $X=\left(x_{i j}\right)$ is the corresponding matrix of $X$, relative to the basis $\left\{e_{n}\right\}$, then the $(i, j)$ entry for $D_{\varepsilon} X-X D_{\varepsilon}$ is $\left(\lambda_{i}-\lambda_{j}\right) x_{i j}$. Similarly the $(i, j)$ entry for $f\left(D_{\varepsilon}\right) X-X f\left(D_{\varepsilon}\right)$ is $\left(f\left(\lambda_{i}\right)-f\left(\lambda_{j}\right)\right) x_{i j}$. Since

$$
\left\|D_{\varepsilon} X-X D_{\varepsilon}\right\|_{2}^{2}=\sum_{i, j}\left|\left(\lambda_{i}-\lambda_{j}\right) x_{i j}\right|^{2}
$$

and

$$
\left\|f\left(D_{\varepsilon}\right) X-X f\left(D_{\varepsilon}\right)\right\|_{2}^{2}=\sum_{i, j}\left|\left(f\left(\lambda_{i}\right)-f\left(\lambda_{j}\right)\right) x_{i j}\right|^{2},
$$

Received by the editors January 18, 1983 and, in revised form, September 21, 1984.

1980 Mathematics Subject Classification. Primary 47B10, 47B15.

Key words and phrases. Lipschitz function, Hilbert-Schmidt operator, normal operator. 
it follows that

$$
\left\|f\left(D_{\varepsilon}\right) X-X f\left(D_{\varepsilon}\right)\right\|_{2} \leqslant k\left\|D_{\varepsilon} X-X D_{\varepsilon}\right\|_{2} .
$$

Next, let $N=\int_{\Omega} z d E(z)$ be the spectral representation of $N$. Then

$$
\begin{aligned}
\|\left(\left(f(N)-f\left(D_{\varepsilon}\right)\right) e_{n} \|^{2}\right. & =\int_{\Omega}\left|f(z)-f\left(\lambda_{n}\right)\right|^{2} d\left\|E(z) e_{n}\right\|^{2} \\
& \leqslant k^{2} \int_{\Omega}\left|z-\lambda_{n}\right|^{2} d\left\|E(z) e_{n}\right\|^{2} \\
& =k^{2}\left\|\left(N-D_{\varepsilon}\right) e_{n}\right\|^{2} .
\end{aligned}
$$

Consequently, $\left\|f(N)-f\left(D_{\varepsilon}\right)\right\|_{2} \leqslant k\left\|N-D_{\varepsilon}\right\|_{2}=k\left\|K_{\varepsilon}\right\|_{2}<\varepsilon k$. Thus $f(N)=f\left(D_{\varepsilon}\right)$ $+C_{\varepsilon}$ with $\left\|C_{\varepsilon}\right\|_{2} \rightarrow 0$ as $\varepsilon \rightarrow 0$. Since

$$
\left|\|N X-X N\|_{2}-\left\|D_{\varepsilon} X-X D_{\varepsilon}\right\|_{2}\right| \leqslant\left\|K_{\varepsilon} X-X K_{\varepsilon}\right\|_{2} \leqslant 2\left\|K_{\varepsilon}\right\|_{2}\|X\|<2\|X\| \varepsilon,
$$

it follows that

$$
\lim _{\varepsilon \rightarrow 0}\left\|D_{\varepsilon} X-X D_{\varepsilon}\right\|_{2}=\|N X-X N\|_{2} \text {. }
$$

Similarly we have $\lim _{\varepsilon \rightarrow 0}\left\|f\left(D_{\varepsilon}\right) X-X f\left(D_{\varepsilon}\right)\right\|_{2}=\|f(N) X-X f(N)\|_{2}$. The required result now follows by letting $\varepsilon \rightarrow 0$ in (*) above.

An alternative proof of the Theorem which was suggested by Professor Ando can be found in the author's doctoral thesis [8].

Corollary 1 (Fuglede's TheOREM MOdulo $C_{2}$ [11]). Let $N$ be a normal operator. Then $\|N X-X N\|_{2}=\left\|N^{*} X-X N^{*}\right\|_{2}$ for all $X \in B(H)$.

Proof. Apply the Theorem to the function $f(z)=\bar{z}$.

S. K. Berberian's trick allows us to extend the Theorem as follows.

Corollary 2. Let $N$ and $M$ be normal operators and let $f$ be a function defined on the union of the spectra of $N$ and $M$. If $|f(z)-f(w)| \leqslant k|z-w|$ for all $z, w \in \sigma(N)$ $\cup \sigma(M)$ and some positive constant $k$, then $\|f(N) X-X f(M)\|_{2} \leqslant k\|N X-X M\|_{2}$ for all $X \in B(H)$. In particular, $\|f(N)-f(M)\|_{2} \leqslant k\|N-M\|_{2}$.

Proof. Define operators $L$ and $Y$ on the space $H \oplus H$ by

$$
L=\left|\begin{array}{cc}
N & 0 \\
0 & M
\end{array}\right|, \quad Y=\left|\begin{array}{cc}
0 & X \\
0 & 0
\end{array}\right| .
$$

Then

$$
\|N X-X M\|_{2}=\|L Y-Y L\|_{2}
$$

and

$$
\|f(N) X-X f(M)\|_{2}=\|f(L) Y-Y f(L)\|_{2} .
$$

Application of the Theorem to $L$ and $Y$ gives the required result.

The special case where $X=I$ in Corollary 2 is of particular interest in perturbation theory of linear operators (see [4-6]). Using the theory of Stieltjes double operator integrals, M. S. Birman and M. Z. Solomyak proved [3, Theorem 11] that if 
$U$ and $V$ are unitary operators and $f$ is a function defined on the unit circle, whose derivative $f^{\prime}$ is Lipschitz of order $\alpha>0$, then $U-V \in C_{2}$ implies that $f(U)-f(V)$ $\in C_{2}$. The special case where $f(z)=|z|$ in Corollary 2 above is also of great importance in the study of quasi-equivalence of quasi-free states of canonical commutation relations (see [2] and the references there).

For $T \in B(H)$, let the absolute value $|T|$ of $T$ be defined as $\left(T^{*} T\right)^{1 / 2}$. H. Araki and S. Yamagami proved [2, Theorem 1] that for any two operators $A$ and $B$ in $B(H),\||A|-|B|\|_{2} \leqslant \sqrt{2}\|A-B\|_{2}$, and they remarked that $\sqrt{2}$ is the best possible coefficient for a general $A$ and $B$. However, if $A$ and $B$ are restricted to be selfadjoint, then the best coefficient is 1 instead of $\sqrt{2}$.

We conclude the paper with the following extension of the selfadjoint case.

COROLlaRY 3. Let $N$ and $M$ be normal operators. Then $\||N|-|M|\|_{2} \leqslant\|N-M\|_{2}$.

Proof. Apply Corollary 2 to the function $f(z)=|z|$.

\section{REFERENCES}

1. W. O. Amrein and D. B. Pearson, Commutators of Hilbert-Schmidt type and the scattering cross section, Ann. Inst. H. Poincaré Sect. A (N.S.) 30 (1979), 89-107.

2. H. Araki and S. Yamagami, An inequality for Hilbert-Schmidt norm, Comm. Math. Phys. 81 (1981), 89-96.

3. M. S. Birman and M. Z. Solomyak, Stieltjes double-integral, Topics in Mathematical Physics, Vol. 1, Consultants Bureau, New York, 1967.

4. Yu. B. Farforovskaya, Example of a Lipschitz function of self-adjoint operators that gives a nonnuclear increment under a nuclear perturbation, J. Soviet Math. 4 (1975), 426-433.

5. __ An estimate of the difference $f(B)-f(A)$ in the class $\gamma_{p}$, J. Soviet Math. 8 (1977), 146-148.

6. (1980), 1133-1149.

7. I. C. Gohberg and M. G. Krein, Introduction to the theory of linear nonself-adjoint operators, Transl. Math. Monographs, Vol. 18, Amer. Math. Soc., Providence, R. I., 1969.

8. F. Kittaneh, Commutators of $C_{p}$ type, $\mathrm{Ph}$. D. Thesis, Indiana Univ., 1982.

9. R. Schatten, Norm ideals of completely continuous operators, Springer-Verlag, Berlin, 1960.

10. D. Voiculescu, Some results on norm ideal perturbations of Hilbert space operators, J. Operator Theory 2 (1979), 3-37.

11. G. Weiss, The Fuglede commutativity theorem modulo the Hilbert-Schmidt class and generating functions for matrix operators.II, J. Operator Theory 5 (1981), 3-16.

Department of Mathematics, United Arab Emirates University, Al-Ain, United Arab EMIRATES 